\title{
Anisotropic Besov Spaces and Approximation Numbers of Traces on Related Fractal Sets
}

\author{
Erika TAMÁSI \\ Mathematical Institute \\ Friedrich-Schiller-University Jena \\ D-07737 Jena - Germany \\ tamasi@minet.uni-jena.de
}

Received: December 25, 2005

Accepted: January 13, 2006

\begin{abstract}
This paper deals with approximation numbers of the compact trace operator of an anisotropic Besov space into some $L_{p}$-space,

$$
\operatorname{tr}_{\Gamma}: B_{p p}^{s, a}\left(\mathbb{R}^{n}\right) \hookrightarrow L_{p}(\Gamma), \quad s>0, \quad 1<p<\infty,
$$

where $\Gamma$ is an anisotropic $d$-set, $0<d<n$. We also prove homogeneity estimates, a homogeneous equivalent norm and the localization property in $B_{p p}^{s, a}$.

Key words: anisotropic function spaces, fractals, approximation numbers, traces.

2000 Mathematics Subject Classification: 46E35, 42B35, 42C40.
\end{abstract}

\section{Introduction}

The theory of the anisotropic spaces has been developed from the very beginning parallel to the theory of isotropic function spaces. We refer in particular to the Russian school and works of S. M. Nikol'skiı̌, O. V. Besov, V. P. Il'in [1,11].

Let $1<p<\infty$ and $\left(s_{1}, \ldots, s_{n}\right)$ be an $n$ - tuple of natural numbers. Then

$$
W_{p}^{s, a}\left(\mathbb{R}^{n}\right)=\left\{f \in \mathcal{S}^{\prime}\left(\mathbb{R}^{n}\right):\left\|f\left|L_{p}\left(\mathbb{R}^{n}\right)\left\|+\sum_{k=1}^{n}\right\| \frac{\partial^{s_{k}} f}{\partial x_{k}^{s_{k}}}\right| L_{p}\left(\mathbb{R}^{n}\right)\right\|<\infty\right\}
$$

is the classical anisotropic Sobolev space on $\mathbb{R}^{n}$. It is obvious that unlike in case of the usual (isotropic) Sobolev space $\left(s_{1}=\cdots=s_{n}\right)$ the smoothness properties of an 
element from $W_{p}^{s, a}\left(\mathbb{R}^{n}\right)$ depend on the chosen direction in $\mathbb{R}^{n}$. The number $s$, defined by

$$
\frac{1}{s}=\frac{1}{n}\left(\frac{1}{s_{1}}+\cdots+\frac{1}{s_{n}}\right)
$$

is usually called the mean smoothness, and $a=\left(a_{1}, \ldots, a_{n}\right)$,

$$
a_{1}=\frac{s}{s_{1}}, \ldots, a_{n}=\frac{s}{s_{n}}
$$

characterizes the anisotropy. Similar to the isotropic situation the more general anisotropic Bessel potential spaces (fractional Sobolev spaces) $H_{p}^{s, a}\left(\mathbb{R}^{n}\right)$, where $1<p<\infty, s \in \mathbb{R}$ and $a=\left(a_{1}, \ldots, a_{n}\right)$ is a given anisotropy, fit in the scales of anisotropic Besov spaces $B_{p q}^{s, a}\left(\mathbb{R}^{n}\right)$, and anisotropic Triebel-Lizorkin spaces $F_{p q}^{s, a}\left(\mathbb{R}^{n}\right)$, respectively. It is well known that this theory has a more or less complete counterpart to the basic facts (definitions, description via differences and derivatives, elementary properties, embeddings for different metrics, interpolation) of isotropic spaces $B_{p q}^{s}\left(\mathbb{R}^{n}\right)$ and $F_{p q}^{s}\left(\mathbb{R}^{n}\right)$. We shall use the Fourier-analytical definition of $B_{p q}^{s, a}\left(\mathbb{R}^{n}\right)$, $F_{p q}^{s, a}\left(\mathbb{R}^{n}\right)$, where any function $f \in \mathcal{S}^{\prime}\left(\mathbb{R}^{n}\right)$ is decomposed in a sum of entire analytic functions $\left(\varphi_{j} \hat{f}\right)^{\vee}$ and this decomposition, measured in $\ell_{q}$ and $L_{p}\left(\mathbb{R}^{n}\right)$, respectively, is used to introduce the spaces. This concept goes back to $[15,16]$, see $[13$, chapter 4$]$.

Our main aim in the present paper is to prove an anisotropic counterpart to the isotropic results, see [21].

As a first goal of this paper we define the anisotropic $d$-set as follows:

Let $0<d<n, a$ an anisotropy. Then $\Gamma \subset \mathbb{R}^{n}$ is called an anisotropic $d$-set if there exists a positive Radon measure $\mu$ with $\operatorname{supp} \mu=\Gamma$ and

$$
\mu\left(B^{a}(\gamma, r)\right) \sim r^{d}, \quad 0<r<1,
$$

where $B^{a}(\gamma, r)=\left\{y \in \mathbb{R}^{n}:|y-\gamma|_{a} \leq r\right\}$ is an anisotropic ball and $\gamma \in \Gamma$.

We study the existence and properties of the trace operator $\operatorname{tr}_{\Gamma}$,

$$
\operatorname{tr}_{\Gamma}: B_{p p}^{s, a}\left(\mathbb{R}^{n}\right) \hookrightarrow L_{p}(\Gamma)
$$

where $\Gamma$ is an anisotropic $d-$ set. It turns out that $\operatorname{tr}_{\Gamma}$ according to (1) exists if, and is compact if

$$
\sum_{j \in \mathbb{N}_{0}} 2^{-j p^{\prime}\left(s-\frac{n}{p}\right)} \mu_{j}^{p^{\prime}-1}<\infty, \quad \frac{1}{p}+\frac{1}{p^{\prime}}=1,
$$

where $\mu_{j}=\sup _{m \in \mathbb{Z}^{n}} \mu\left(Q_{j m}^{a}\right)$, and $Q_{j m}^{a}$ are rectangles centered at $2^{-j a} m$ and with side length $2^{-j a_{1}}, \ldots, 2^{-j a_{n}}$. If we can strengthen $(2)$ by

$$
\sum_{j \geq J} 2^{-j p^{\prime}\left(s-\frac{n}{p}\right)} \mu_{j}^{p^{\prime}-1} \sim 2^{-J p^{\prime}\left(s-\frac{n}{p}\right)} \mu_{J}^{p^{\prime}-1}, \quad J \in \mathbb{N}_{0},
$$


then one obtains for the approximation numbers $a_{k}$ of the compact operator $\operatorname{tr}_{\Gamma}$ according to (1)

$$
a_{k}\left(\operatorname{tr}_{\Gamma}: B_{p p}^{s, a}\left(\mathbb{R}^{n}\right) \hookrightarrow L_{p}(\Gamma)\right) \sim k^{\frac{1}{d}\left(\frac{n}{p}-s\right)-\frac{1}{p}}, \quad \frac{n}{p} \geq s>\frac{n-d}{p},
$$

as in the isotropic case, see [21]. In order to show the above result we prove, in addition, some important properties of spaces $B_{p q}^{s, a}\left(\mathbb{R}^{n}\right)$, with $0<p \leq \infty, 0<q \leq \infty$, and $s>n\left(\frac{1}{p}-1\right)_{+}$, which might be of self-contained interest:

(i) We obtain the homogeneity estimate

$$
\left\|f(R \cdot)\left|B_{p q}^{s, a}\left(\mathbb{R}^{n}\right)\left\|\leq c R^{s-\frac{n}{p}}\right\| f\right| B_{p q}^{s, a}\left(\mathbb{R}^{n}\right)\right\|
$$

for all $f \in B_{p q}^{s, a}\left(\mathbb{R}^{n}\right)$ and $R \geq 1$.

(ii) We show that

$$
\left\|(\varphi \hat{f})^{\vee} \mid L_{p}\right\|+\left(\sum_{j=-\infty}^{\infty} 2^{j s q}\left\|\left(\varphi_{j}^{a} \hat{f}\right)^{\vee} \mid L_{p}\right\|^{q}\right)^{1 / q}
$$

and

$$
\left\|f \mid L_{p}\right\|+\left(\sum_{j=-\infty}^{\infty} 2^{j s q}\left\|\left(\varphi_{j}^{a} \hat{f}\right)^{\vee} \mid L_{p}\right\|^{q}\right)^{1 / q}
$$

are equivalent quasi-norms in $B_{p q}^{s, a}\left(\mathbb{R}^{n}\right)$.

(iii) Finally we prove the localization property of $B_{p p}^{s, a}\left(\mathbb{R}^{n}\right)$, that is

$$
2^{j\left(s-\frac{n}{p}\right)}\left(\sum_{k \in \mathbb{Z}^{n}}\left|c_{k}\right|^{p}\right)^{1 / p}\left\|f\left|B_{p p}^{s, a}\left(\mathbb{R}^{n}\right)\|\sim\| f_{j}^{a}\right| B_{p p}^{s, a}\left(\mathbb{R}^{n}\right)\right\|,
$$

where

$$
f_{j}^{a}(x)=\sum_{k \in \mathbb{Z}^{n}} c_{k} f\left(2^{(j+1) a}\left(x-x_{j, k}^{a}\right)\right), \quad c_{k} \in \mathbb{C}, \quad j \in \mathbb{N},
$$

and $f$ is a product of one-dimensional functions

$$
f\left(2^{(j+1) a}\left(x-x_{j, k}^{a}\right)\right)=\prod_{m=1}^{n} f_{m}\left(2^{(j+1) a_{m}}\left(x_{m}-2^{-j a_{m}} k_{m}\right)\right) .
$$

The plan of the paper is the following. In the second section we give the definition and some important properties of anisotropic Besov spaces. In section 3 we introduce the anisotropic $d$-set and we formulate our main result. In the last section we collect the proofs and give a description of the wavelet frames according to [9]. 


\section{Anisotropic Besov spaces}

\subsection{General notation}

As usual, $\mathbb{R}^{n}$ denotes the $n$-dimensional real Euclidean space, $\mathbb{N}$ the collection of all natural numbers, $\mathbb{N}_{0}=\mathbb{N} \cup\{0\}, \mathbb{C}$ stands for the complex numbers, and $\mathbb{Z}^{n}$ means the lattice of all points in $\mathbb{R}^{n}$ with integer-valued components. We use the equivalence " $\sim$ " in $\varphi(x) \sim \psi(x)$ always to mean that there are two positive numbers $c_{1}$ and $c_{2}$ such that

$$
c_{1} \varphi(x) \leq \psi(x) \leq c_{2} \varphi(x)
$$

for all admitted values of $x$, where $\varphi, \psi$ are non-negative functions. If $a \in \mathbb{R}$ then $a_{+}:=\max (a, 0)$. Let $\alpha=\left(\alpha_{1}, \ldots, \alpha_{n}\right) \in \mathbb{N}_{0}^{n}$ be a multi-index, then

$$
|\alpha|=\alpha_{1}+\cdots+\alpha_{n}, \quad \alpha !=\alpha_{1} ! \cdots \alpha_{n} !, \quad \alpha \in \mathbb{N}_{0}^{n},
$$

the derivatives $D^{\alpha}$ have the usual meaning, $x^{\alpha}$ means $x^{\alpha}=x_{1}^{\alpha_{1}} \cdots x_{n}^{\alpha_{n}}$ for $x=$ $\left(x_{1}, \ldots, x_{n}\right) \in \mathbb{R}^{n}$, and $\alpha \gamma=\alpha_{1} \gamma_{1}+\cdots+\alpha_{n} \gamma_{n}, \gamma \in \mathbb{R}^{n}$, stands for the scalar product in $\mathbb{R}^{n}$.

Given two quasi-Banach spaces $X$ and $Y$, we write $X \hookrightarrow Y$ if $X \subset Y$ and the natural embedding of $X$ in $Y$ is continuous. All unimportant positive constants will be denoted by $c$, occasionally with additional subscripts within the same formula. We shall mainly deal with function spaces on $\mathbb{R}^{n}$; so for convenience we shall usually omit the " $\mathbb{R}^{n}$ " from their notation, if there is no danger of confusion.

\subsection{Definitions}

Let $a=\left(a_{1}, \ldots, a_{n}\right)$ be a fixed $n$-tuple of positive numbers with $a_{1}+\cdots+a_{n}=n$, then we call $a$ an anisotropy. We shall denote $a_{\min }=\min \left\{a_{i}: 1 \leq i \leq n\right\}$ and $a_{\max }=\max \left\{a_{i}: 1 \leq i \leq n\right\}$. If $a=(1, \ldots, 1)$ we speak about the "isotropic case".

The action of $t \in[0, \infty)$ on $x \in \mathbb{R}^{n}$ is defined by the formula

$$
t^{a} x=\left(t^{a_{1}} x_{1}, \ldots, t^{a_{n}} x_{n}\right)
$$

For $t>0$ and $s \in \mathbb{R}$ we put $t^{s a} x=\left(t^{s}\right)^{a} x$. In particular we write $t^{-a} x=\left(t^{-1}\right)^{a} x$ and $2^{-j a} x=\left(2^{-j}\right)^{a} x$.

Definition 2.1. An anisotropic distance function is a continuous function $u: \mathbb{R}^{n} \rightarrow \mathbb{R}$ with the properties $u(x)>0$ if $x \neq 0$ and $u\left(t^{a} x\right)=t u(x)$ for all $t>0$ and all $x \in \mathbb{R}^{n}$.

Remark 2.2. It is easy to see that $u_{\lambda}: \mathbb{R}^{n} \rightarrow \mathbb{R}$ defined by

$$
u_{\lambda}(x)=\left(\sum_{i=1}^{n}\left|x_{i}\right|^{\frac{\lambda}{a_{i}}}\right)^{1 / \lambda}
$$

is an anisotropic distance function for every $0<\lambda<\infty, u_{2}$ is usually called the anisotropic distance of $x$ to the origin, see [13, 4.2.1]. It is well known, see [3, 1.2.3] 
and $[22,1.4]$, that any two anisotropic distance functions $u$ and $u^{\prime}$ are equivalent (in the sense that there exist constants $c, c^{\prime}>0$ such that $c u(x) \leq u^{\prime}(x) \leq c^{\prime} u(x)$ for all $x \in \mathbb{R}^{n}$ ) and that if $u$ is an anisotropic distance function there exists a constant $c>0$ such that $u(x+y) \leq c(u(x)+u(y))$ for all $x, y \in \mathbb{R}^{n}$. We are interested to use smooth anisotropic distance functions. Note that for appropriate values of $\lambda$ one can obtain arbitrary (finite) smoothness of the function $u_{\lambda}$ from (4), cf. [3, 1.2.4]. A standard method concerning the construction of anisotropic distance functions in $C^{\infty}\left(\mathbb{R}^{n} \backslash\{0\}\right)$ was given in [14].

For $x=\left(x_{1}, \ldots, x_{n}\right) \in \mathbb{R}^{n}, x \neq 0$, let $|x|_{a}$ be the unique positive number $t$ such that

$$
\frac{x_{1}^{2}}{t^{2 a_{1}}}+\cdots+\frac{x_{n}^{2}}{t^{2 a_{n}}}=1
$$

and let $|0|_{a}=0$; then $|\cdot|_{a}$ is an anisotropic distance function in $C^{\infty}\left(\mathbb{R}^{n} \backslash\{0\}\right)$, see $[22,1.4 / 3,8]$. Plainly, $|x|_{a}$ is in the isotropic case the Euclidean distance of $x$ to the origin.

Before introducing the function spaces under consideration we need to recall some notation. By $\mathcal{S}$ we denote the Schwartz space of all complex-valued, infinitely differentiable and rapidly decreasing functions on $\mathbb{R}^{n}$ and by $\mathcal{S}^{\prime}$ the dual space of all tempered distributions on $\mathbb{R}^{n}$. Furthermore, $L_{p}$ with $0<p \leq \infty$, stands for the usual quasi-Banach space with respect to the Lebesgue measure, quasi-normed by

$$
\left\|f \mid L_{p}\right\|:=\left(\int_{\mathbb{R}^{n}}|f(x)|^{p} d x\right)^{1 / p}
$$

with the obvious modification if $p=\infty$. If $\varphi \in \mathcal{S}$ then

$$
\hat{\varphi}(\xi) \equiv(\mathcal{F} \varphi)(\xi):=(2 \pi)^{-n / 2} \int_{\mathbb{R}^{n}} e^{-i x \xi} \varphi(x) d x, \quad \xi \in \mathbb{R}^{n},
$$

denotes the Fourier transform of $\varphi$. As usual, $\mathcal{F}^{-1} \varphi$ or $\check{\varphi}$ stands for the inverse Fourier transform, given by the right-hand side of (5) with $i$ in place of $-i$. Here $x \xi$ denotes the scalar product in $\mathbb{R}^{n}$. Both $\mathcal{F}$ and $\mathcal{F}^{-1}$ are extended to $\mathcal{S}^{\prime}$ in the standard way. Let $\varphi \in \mathcal{S}$ be such that

$$
\varphi(x)=1 \quad \text { if } \quad|x|_{a} \leq 1 \text { and } \operatorname{supp} \varphi \subset\left\{x \in \mathbb{R}^{n}:|x|_{a} \leq 2\right\},
$$

and for each $j \in \mathbb{N}$ let

$$
\varphi_{j}^{a}(x):=\varphi\left(2^{-j a} x\right)-\varphi\left(2^{(-j+1) a} x\right), \quad x \in \mathbb{R}^{n} .
$$

Then the sequence $\left(\varphi_{j}^{a}\right)_{j=0}^{\infty}$, with $\varphi_{0}=\varphi$, forms a smooth anisotropic dyadic resolution of unity, cf. $[13,4.2]$. Let $f \in \mathcal{S}^{\prime}$, then the compact support of $\varphi_{j}^{a} \hat{f}$ implies by the Paley-Wiener-Schwartz theorem that $\left(\varphi_{j}^{a} \hat{f}\right)^{\vee}$ is an entire analytic function on $\mathbb{R}^{n}$. 
Definition 2.3. Assume $0<p \leq \infty, 0<q \leq \infty, s \in \mathbb{R}, a$ an anisotropy, and $\left(\varphi_{j}^{a}\right)_{j=0}^{\infty}$ a smooth anisotropic dyadic resolution of unity. Then

$$
B_{p q}^{s, a}=\left\{f \in \mathcal{S}^{\prime}:\left\|f \mid B_{p q}^{s, a}\right\|_{\varphi}=\left(\sum_{j=0}^{\infty} 2^{j s q}\left\|\left(\varphi_{j}^{a} \hat{f}\right)^{\vee} \mid L_{p}\right\|^{q}\right)^{1 / q}<\infty\right\}
$$

(with the usual modification if $q=\infty$ ).

Note that there is a parallel definition for spaces of type $F_{p q}^{s, a}, 0<p<\infty$, $0<q \leq \infty, s \in \mathbb{R}, a$ an anisotropy, when interchanging the order of $\ell_{q^{-}}$and $L_{p^{-}}$-quasinorms in (8). It is obvious that the quasi-norm (8) depends on the chosen system $\left(\varphi_{j}^{a}\right)_{j \in \mathbb{N}_{0}}$, but not the space $B_{p q}^{s, a}$ (in the sense of equivalent quasi-norms); therefore we omit in our notation the subscript $\varphi$ in the sequel. It is well-known that $B_{p q}^{s, a}$ are quasi-Banach spaces (Banach spaces if $p \geq 1$ and $q \geq 1$ ), and, as in the isotropic case, $\mathcal{S} \hookrightarrow B_{p q}^{s, a} \hookrightarrow \mathcal{S}^{\prime}$ for all admissible values of $p, q, s$, see [17, 2.3.3]. If $s \in \mathbb{R}$ and $0<p<\infty, 0<q<\infty$ then $\mathcal{S}$ is dense in $B_{p q}^{s, a}$, see $[3,1.2 .10 ; 22,3.5]$. Note that we indicated the only (formal) difference to the isotropic counterparts of (8) by the additional superscript at the smooth anisotropic dyadic resolution of unity $\left(\varphi_{j}^{a}\right)_{j=0}^{\infty}$.

Remark 2.4. A systematic treatment of the theory of (isotropic) $B_{p q}^{s}$ (and $F_{p q}^{s}$ ) spaces may be found in the monographs [17-20]; see also [4,12]. A survey on the basic results for the (anisotropic) spaces $B_{p q}^{s, a}$ (and $F_{p q}^{s, a}$ ) is given in $[10,2.1-2.2 ; 13,4.2 .1-4.2 .4]$. In addition to the literature mentioned in our introduction we essentially rely on $[7,8]$ in the sequel.

For convenience, in case of $p=q$ we shall stick to the notation

$$
B_{p}^{s, a}=B_{p p}^{s, a} \quad \text { where } \quad 0<p \leq \infty, \quad s \in \mathbb{R},
$$

in the sequel.

\subsection{Properties}

Our aim is to prove some new and important properties of anisotropic Besov spaces and thus to complement results in $[2,7,13]$. This also serves as preparation for our main results in section 3 .

Let $\varphi \in \mathcal{S}$ as in section 2.2. In particular we have (6). We extend the definition of $\varphi_{j}^{a}$ from (7) to all integers $j$. It should be noted that $\varphi_{0}^{a}$ has now a different meaning as in 2.2 , i.e., for $f \in \mathcal{S}^{\prime}$ then we have that

$$
f=(\varphi \hat{f})^{\vee}+\sum_{j=1}^{\infty}\left(\varphi_{j}^{a} \hat{f}\right)^{\vee} \quad\left(\text { convergence in } \mathcal{S}^{\prime}\right) .
$$

As usual, let $\sigma_{p}=n\left(\frac{1}{p}-1\right)_{+}, \quad 0<p \leq \infty$. 
Theorem 2.5. Let $0<p \leq \infty, 0<q \leq \infty, s>\sigma_{p}$ and a an anisotropy, then

$$
\left\|(\varphi \hat{f})^{\vee} \mid L_{p}\right\|+\left(\sum_{j=-\infty}^{\infty} 2^{j s q}\left\|\left(\varphi_{j}^{a} \hat{f}\right)^{\vee} \mid L_{p}\right\|^{q}\right)^{1 / q}
$$

and

$$
\left\|f \mid L_{p}\right\|+\left(\sum_{j=-\infty}^{\infty} 2^{j s q}\left\|\left(\varphi_{j}^{a} \hat{f}\right)^{\vee} \mid L_{p}\right\|^{q}\right)^{1 / q}
$$

(modification if $q=\infty$ ) are equivalent quasi-norms in $B_{p q}^{s, a}$.

Remark 2.6. The quasi-norms of type (10), (11) have a continuous counterpart. We introduce $\rho^{a}(t \xi)=\varphi\left(t^{a} \xi\right)-\varphi\left((2 t)^{a} \xi\right)$ where $t>0$. Then the counterpart of (10) reads as follows:

Let $0<p \leq \infty, 0<q \leq \infty, s>\sigma_{p}$ and $a$ an anisotropy, then

$$
\left\|f \mid L_{p}\right\|+\left(\int_{0}^{\infty} t^{-s q}\left\|\left(\rho^{a}(t \cdot) \hat{f}\right)^{\vee} \mid L_{p}\right\|^{q} \frac{d t}{t}\right)^{1 / q}
$$

(modification if $q=\infty$ ) is an equivalent quasi-norm in $B_{p q}^{s, a}$.

The proof is given in section 4.1. Now we can extend the well-known homogeneity estimate for $B_{p q}^{s}$ (see [17, Prop. 3.4.1]) to anisotropic spaces.

Proposition 2.7. Let $0<p \leq \infty, 0<q \leq \infty, s>\sigma_{p}$ and $a$ an anisotropy. There exists a constant $c>0$ such that for all $R \geq 1$,

$$
\left\|f(R \cdot)\left|B_{p q}^{s, a}\left\|\leq c R^{s-\frac{n}{p}}\right\| f\right| B_{p q}^{s, a}\right\| \quad \text { for all } f \in B_{p q}^{s, a} .
$$

The proof of this proposition is contained in section 4.2 .

Our next aim is to extend the localization property, see [4, 2.3.2], to anisotropic spaces.

Let $x_{j, k}^{a}=2^{-j a} k$ with $k \in \mathbb{Z}^{n}$ and $j \in \mathbb{N}$. Let $f \in \mathcal{S}^{\prime}$ with

$$
\operatorname{supp} f \subset Q_{b}^{a}=\left\{x \in \mathbb{R}^{n}: x=\left(x_{1}, x_{2}, \ldots, x_{n}\right),|x|_{a}<b\right\}
$$

where $b>0$ and $b \leq \frac{1}{4}\left(2^{a_{\min }}+1\right)$. Let

$$
f_{j}^{a}(x)=\sum_{k \in \mathbb{Z}^{n}} c_{k} f\left(2^{(j+1) a}\left(x-x_{j, k}^{a}\right)\right), \quad c_{k} \in \mathbb{C}, \quad j \in \mathbb{N}
$$

where $f$ is a product of one-dimensional functions,

$$
f\left(2^{(j+1) a}\left(x-x_{j, k}^{a}\right)\right)=\prod_{m=1}^{n} f_{m}\left(2^{(j+1) a_{m}}\left(x_{m}-2^{-j a_{m}} k_{m}\right)\right)
$$

and $f_{1}(y)=\cdots=f_{n}(y)$ where $y \in \mathbb{R}$. 
Theorem 2.8. Let $s>\sigma_{p}, 0<p \leq \infty$, a an anisotropy and $0<b \leq \frac{1}{4}\left(2^{a_{\min }}+1\right)$. There exist two constants $c^{\prime}>0$ and $c^{\prime \prime}>0$ such that for all $f \in B_{p}^{s, a}$ with $\operatorname{supp} f \subset$ $Q_{b}^{a}$ and all $j \in \mathbb{N}$ and all $f_{j}^{a}$ given by (15)

$$
c^{\prime}\left\|f_{j}^{a}\left|B_{p}^{s, a}\left\|\leq 2^{j\left(s-\frac{n}{p}\right)}\left(\sum_{k \in \mathbb{Z}^{n}}\left|c_{k}\right|^{p}\right)^{1 / p}\right\| f\right| B_{p}^{s, a}\right\| \leq c^{\prime \prime}\left\|f_{j}^{a} \mid B_{p}^{s, a}\right\| .
$$

We prove this result in section 4.3.

\section{Traces and approximation numbers}

\subsection{General measures}

Let $\mu$ be a positive Radon measure in $\mathbb{R}^{n}$ with compact support

$$
\Gamma=\operatorname{supp} \mu, \quad 0<\mu\left(\mathbb{R}^{n}\right)<\infty, \quad|\Gamma|=0,
$$

where $|\Gamma|$ is the Lebesgue measure of $\Gamma$. For $1 \leq p<\infty$ we denote by $L_{p}(\Gamma)=L_{p}(\Gamma, \mu)$ the usual complex Banach space, normed by

$$
\left\|f \mid L_{p}(\Gamma, \mu)\right\|=\left(\int_{\mathbb{R}^{n}}|f(x)|^{p} \mu(d x)\right)^{1 / p}=\left(\int_{\Gamma}|f(\gamma)|^{p} \mu(d \gamma)\right)^{1 / p} .
$$

Since $\mu$ is Radon, $S\left(\mathbb{R}^{n}\right) \mid \Gamma$ is dense in $L_{p}(\Gamma)$. If $\varphi \in \mathcal{S}$ then $\operatorname{tr}_{\Gamma} \varphi=\varphi \mid \Gamma$ makes sense pointwise. If $1<p<\infty, 0<q \leq \infty$ and $s>0$ then the embedding $\operatorname{tr}_{\Gamma} B_{p q}^{s, a} \hookrightarrow L_{p}(\Gamma)$ must be understood as follows: there exists a positive number $c>0$ such that for any $\varphi \in \mathcal{S}$

$$
\left\|\operatorname{tr}_{\Gamma} \varphi\left|L_{p}(\Gamma)\|\leq c\| \varphi\right| B_{p q}^{s, a}\right\| .
$$

Since $\mathcal{S}$ is dense in $B_{p q}^{s, a}$ for $0<p, q<\infty$ this inequality can be extended by completion to any $f \in B_{p q}^{s, a}$ and the resulting function is denoted by $\operatorname{tr}_{\Gamma} f$ and the independence of $\operatorname{tr}_{\Gamma} f$ from the approximating sequence is shown in the standard way.

In the sequel, we only consider the case $p=q$. We proceed in a way similar to [21], dealing with the isotropic case. Let $Q_{j m}^{a}$ be the rectangles in $\mathbb{R}^{n}$ with side length $2^{-j a_{1}}, \ldots, 2^{-j a_{n}}$ and centered at $2^{-j a} m$ where $m \in \mathbb{Z}^{n}$ and $j \in \mathbb{N}_{0}$. Let

$$
\mu_{j}=\sup _{m \in \mathbb{Z}^{n}} \mu\left(Q_{j m}^{a}\right), \quad j \in \mathbb{N}_{0} .
$$

Proposition 3.1. Let

$$
1<p<\infty, \quad \frac{1}{p}+\frac{1}{p^{\prime}}=1, \quad s>0 .
$$

Let $\mu$ be the Radon measure in $\mathbb{R}^{n}$ with

$$
\Gamma=\operatorname{supp} \mu \text { compact }, \quad 0<\mu\left(\mathbb{R}^{n}\right)<\infty, \quad|\Gamma|=0,
$$


and

$$
\sum_{j \in \mathbb{N}_{0}} 2^{-j p^{\prime}\left(s-\frac{n}{p}\right)} \mu_{j}^{p^{\prime}-1}<\infty \quad \text { where } \quad \mu_{j}=\sup _{m \in \mathbb{Z}^{n}} \mu\left(Q_{j m}^{a}\right)
$$

Then

$$
\operatorname{tr}_{\Gamma}: B_{p}^{s, a}\left(\mathbb{R}^{n}\right) \hookrightarrow L_{p}(\Gamma)
$$

exists and is compact. Furthermore there is a constant $c$ (depending on $p$ and $s$ ) such that for all measures $\mu$ with (20), (21),

$$
\left\|\operatorname{tr}_{\Gamma}\right\| \leq c\left(\sum_{j \in \mathbb{N}_{0}} 2^{-j p^{\prime}\left(s-\frac{n}{p}\right)} \mu_{j}^{p^{\prime}-1}\right)^{\frac{1}{p^{\prime}}}
$$

The result above is the anisotropic version of [21, Proposition 3]. The proof can be found in section 4.5.

In the following we recall the concept of approximation numbers. Let $A$ and $B$ be two Banach spaces and let $T \in L(A, B)$. Then for any $k \in \mathbb{N}$ the $k$ th approximation number $a_{k}(T)$ of $T$ is given by

$$
a_{k}(T)=\inf \{\|T-L\|: L \in L(A, B), \quad \operatorname{rank} L<k\},
$$

where rank $L$ is the dimension of the range of $L$. These numbers have various properties given in the following lemma.

Lemma 3.2. Let $A$ and $B$ be two Banach spaces and let $T, S \in L(A, B)$.

(i) $\|T\|=a_{1}(T) \geq a_{2}(T) \geq \cdots \geq 0$.

(ii) For all $n, m \in \mathbb{N}$,

$$
a_{m+n-1}(S+T) \leq a_{m}(S)+a_{n}(T)
$$

(iii) For all $n, m \in \mathbb{N}$, and $R \in L(B, C)$

$$
a_{m+n-1}(R T) \leq a_{m}(R) a_{n}(T) .
$$

(iv) $a_{n}(T)=0 \Longleftrightarrow \operatorname{rank} T<n$.

This is a well-known result and can be found for instance in $[4,1.3 .1]$ and $[5, \mathrm{II}]$. Let $T=\operatorname{tr}_{\Gamma}$ according to Proposition 3.1. We strengthen (21) by

$$
\sum_{j \geq J} 2^{-j p^{\prime}\left(s-\frac{n}{p}\right)} \mu_{j}^{p^{\prime}-1} \sim 2^{-J p^{\prime}\left(s-\frac{n}{p}\right)} \mu_{J}^{p^{\prime}-1}, \quad J \in \mathbb{N}_{0},
$$

where only the cases $s \leq \frac{n}{p}$ are of interest, otherwise (25) is always satisfied. 
Proposition 3.3. Let

$$
1<p<\infty, \quad \frac{1}{p}+\frac{1}{p^{\prime}}=1, \quad s>0 .
$$

Let $\mu$ be a Radon measure in $\mathbb{R}^{n}$ with (20) and (25). Let $a_{k}=a_{k}\left(\operatorname{tr}_{\Gamma}\right)$ be the approximation numbers of the compact operator $\operatorname{tr}_{\Gamma}$ in (22). There are two positive numbers $c$ and $c^{\prime}$ such that

$$
a_{c 2^{n J}} \leq c^{\prime} 2^{-J\left(s-\frac{n}{p}\right)} \mu_{J}^{\frac{1}{p}}, \quad J \in \mathbb{N}_{0},
$$

where $c 2^{n J}$ is always assumed to be a natural number.

We prove the proposition in section 4.6.

\subsection{Anisotropic d-sets in $\mathbb{R}^{n}$}

We consider special measures $\mu$ and assume $\Gamma=\operatorname{supp} \mu$ for some measure according to section 3.1 , in particular with (18), now. Let again $a=\left(a_{1}, \ldots, a_{n}\right)$ be a given anisotropy.

Definition 3.4. Let $0<d<n$. Then $\Gamma \subset \mathbb{R}^{n}$ is called an anisotropic $d$-set if

$$
\mu\left(B^{a}(\gamma, r)\right) \sim r^{d}, \quad 0<r<1,
$$

where $B^{a}(\gamma, r)=\left\{y \in \mathbb{R}^{n}:|y-\gamma|_{a} \leq r\right\}$ and $\gamma \in \Gamma$.

In the following proposition we prove the existence of anisotropic $d$-sets.

Proposition 3.5. For every $0<d<n$ there exists an anisotropic d-set.

Remark 3.6. One can show that our definition for the anisotropic $d$-set is a generalization of Farkas' definition in [8, 3.1].

\subsection{Main assertion}

We are now prepared to formulate our main result.

Theorem 3.7. Let the anisotropic $d$-set $\Gamma$ and $\mu$ be given according to (27), and

$$
0<d<n, \quad 1<p<\infty, \quad \frac{1}{p}+\frac{1}{p^{\prime}}=1, \quad \frac{n}{p} \geq s>\frac{n-d}{p} .
$$

Let $a_{k}=a_{k}\left(\operatorname{tr}_{\Gamma}\right)$ be the approximation numbers of the compact operator $\operatorname{tr}_{\Gamma}$ according to (22). Then there exist numbers $c, c^{\prime}>0$ so that for all $k \in \mathbb{N}$

$$
c k^{\frac{1}{d}\left(\frac{n}{p}-s\right)-\frac{1}{p}} \leq a_{k}\left(\operatorname{tr}_{\Gamma}: B_{p}^{s, a}\left(\mathbb{R}^{n}\right) \hookrightarrow L_{p}(\Gamma)\right) \leq c^{\prime} k^{\frac{1}{d}\left(\frac{n}{p}-s\right)-\frac{1}{p}} .
$$


Remark 3.8. Let $\Gamma$ be the anisotropic $d$-set considered in $[8,3.1]$, see Remark 3.6. Farkas proved in this situation that

$$
e_{k}\left(\operatorname{tr}_{\Gamma}: B_{p_{1} q}^{\delta+\frac{n-d}{p_{1}}, a}\left(\mathbb{R}^{n}\right) \longrightarrow L_{p_{2}}(\Gamma)\right) \sim c k^{-\frac{\delta}{d}}
$$

where $0<p_{1}, p_{2}, q \leq \infty$, and $\delta>0$. Let $p_{1}=p_{2}=q=p$ and $s=\delta+\frac{n-d}{p}$. Then

$$
e_{k}\left(\operatorname{tr}_{\Gamma}: B_{p}^{s, a}\left(\mathbb{R}^{n}\right) \longrightarrow L_{p}(\Gamma)\right) \sim c k^{\frac{1}{d}\left(\frac{n}{p}-s\right)-\frac{1}{p}} .
$$

So we have the same results for the entropy and approximation numbers in the special case $p_{1}=p_{2}$ which is not surprising, but cannot be expected for $p_{1} \neq p_{2}$.

In view of the isotropic result [21, Theorem 2, Remark 9], if we restrict the outcome [21] to the classical example of a compact $d$-set with $0<d<n$, then we have the same result like in the anisotropic setting.

\section{Proofs}

\subsection{Proof of Theorem 2.5}

Proof. We closely follow the proof in $[18,2.3 .3]$ for the isotropic case.

Step 1. We prove that (10) is an equivalent quasi-norm in $B_{p q}^{s, a}$. It is sufficient to show that there exists a constant $c>0$ such that

$$
\left\|\left(\varphi_{j}^{a} \hat{f}\right)^{\vee}\left|L_{p}\left\|\leq c 2^{-j \sigma_{p}}\right\|(\varphi \hat{f})^{\vee}\right| L_{p}\right\|, \quad-j \in \mathbb{N},
$$

holds, because we need to prove that

$$
\left(\sum_{j=-\infty}^{-1} 2^{j s q}\left\|\left(\varphi_{j}^{a} \hat{f}\right)^{\vee} \mid L_{p}\right\|^{q}\right)^{1 / q} \leq c\left\|(\varphi \hat{f})^{\vee} \mid L_{p}\right\|
$$

and this is satisfied if (29) is true. For those $j$ 's we have that $\varphi_{j}^{a}(x)=\varphi_{j}^{a}(x) \varphi(x)$ by the support condition (6) and (7) with $-j \in \mathbb{N}$, and hence

$$
\begin{aligned}
\left\|\left(\varphi_{j}^{a} \hat{f}\right)^{\vee} \mid L_{p}\right\| & =\|\left(\varphi_{j}^{a}\left((\varphi \hat{f})^{\vee}\right)^{)^{\vee}} \mid L_{p} \|\right. \\
& \leq c\left\|\check{\varphi}_{j}^{a}\left|L_{r}\|\|(\varphi \hat{f})^{\vee}\right| L_{p}\right\|, \quad r=\min (1, p),
\end{aligned}
$$

where the inequality comes from the Fourier multiplier assertion for entire analytic functions, $\left\|F^{-1} M F f\left|L_{p}\|\leq\| F^{-1} M\right| L_{\tilde{p}}\right\|\left\|f \mid L_{p}\right\|$ where $\tilde{p}=\min (1, p)$, proved in [17, Proposition 1.5.1]. Elementary calculations show that $\check{\varphi}_{j}^{a}(x)=2^{j n} \check{\varphi}_{0}\left(2^{j a} x\right)$ such that $\left\|\check{\varphi}_{j}^{a}\left|L_{r}\left\|=2^{-j \frac{n}{r}}\right\| \check{\varphi}_{0}\left(2^{j a} \cdot\right)\right| L_{r}\right\| \leq c 2^{-j \frac{n}{r}+j n}$ as $a_{1}+\cdots+a_{n}=n$. By (30) we thus have that

$$
\left\|\left(\varphi_{j}^{a} \hat{f}\right)^{\vee}\left|\mathrm{E}_{p}\left\|\leq 2^{-j n\left(\frac{1}{r}-1\right)}\right\|(\varphi \hat{f})^{\vee}\right| L_{p}\right\|
$$

and we obtain $(29)$ since $\sigma_{p}=n\left(\frac{1}{r}-1\right)$. 
Step 2. We prove that (11) is an equivalent quasi-norm in $B_{p q}^{s, a}$. By our assumption $s>\sigma_{p}$, we may assume that (9) converges not only in $\mathcal{S}^{\prime}$, but also, say almost everywhere in $\mathbb{R}^{n}$. Then we have

$$
\left\|f\left|L_{p}\|\leq c\|(\varphi \hat{f})^{\vee}\right| L_{p}\right\|+c\left(\sum_{j=1}^{\infty}\left\|\left(\varphi_{j}^{a} \hat{f}\right)^{\vee} \mid L_{p}\right\|^{p}\right)^{1 / p}
$$

if $0<p \leq 1$ and a corresponding estimate if $1<p<\infty$. Now (10) and (31) prove that (11) can be estimated from above by $c\left\|f \mid B_{p q}^{s, a}\right\|$. We consider the converse inequality. Because $f$ is a regular distribution we have a.e. that

$$
(\varphi \hat{f})^{\vee}(x)=f(x)+((1-\varphi(\cdot)) \hat{f})^{\vee}(x)=f(x)+\sum_{j=0}^{\infty}\left((1-\varphi(\cdot)) \varphi_{j}^{a}(\cdot) \hat{f}\right)^{\vee}(x) .
$$

By the above-mentioned Fourier multiplier assertion we have

$$
\left\|(\varphi \hat{f})^{\vee}\left|L_{p}\|\leq c\| f\right| L_{p}\right\|+c\left(\sum_{j=0}^{\infty}\left\|\left(\varphi_{j}^{a} \hat{f}\right)^{\vee} \mid L_{p}\right\|^{p}\right)^{1 / p}
$$

if $0<p \leq 1$ and a corresponding estimate if $1<p<\infty$. Now (10) and (32) prove that $\left\|f \mid B_{p q}^{s, a}\right\|$ can be estimated from above by the quasi-norm (11).

\subsection{Proof of Proposition 2.7}

Proof. We closely follow the proof in [4, Prop. 2.3.1] for the isotropic case. Let $\psi=\varphi_{1}$ be the same function as in (7). We have by (12)

$$
\left\|f \mid L_{p}\right\|+\left(\int_{0}^{\infty} t^{-s q}\left\|(\psi(t \cdot) \hat{f})^{\vee} \mid L_{p}\right\|^{q} \frac{d t}{t}\right)^{1 / q}
$$

is an equivalent quasi-norm on $B_{p q}^{s, a}$. Elementary calculation shows that

$$
\begin{aligned}
\left(\psi(t \cdot) f(R \cdot)^{\wedge}(\cdot)\right)^{\vee}(x) & =\left(\psi(t \cdot) \hat{f}\left(R^{-1} \cdot\right)\right)^{\vee}(x) R^{-n} \\
& =(\psi(t(R \cdot)) \hat{f}(\cdot))^{\vee}(R x) .
\end{aligned}
$$

also in the anisotropic case, where $a_{1}+\cdots+a_{n}=n$. From (33), with $f(R x)$ in place of $f(x)$, and (34) we obtain

$$
\begin{aligned}
\left\|f(R \cdot) \mid B_{p q}^{s, a}\right\| & \leq c_{1}\left\|f(R \cdot) \mid L_{p}\right\|+c_{1}\left(\int_{0}^{\infty} t^{-s q}\left\|\mathcal{F}^{-1}(\psi(t \cdot) \mathcal{F}[f(R \cdot)]) \mid L_{p}\right\|^{q} \frac{d t}{t}\right)^{1 / q} \\
& \leq c_{2} R^{-\frac{n}{p}}\left\|f \mid L_{p}\right\|+c_{3} R^{s-\frac{n}{p}}\left(\int_{0}^{\infty} t^{-s q}\left\|\mathcal{F}^{-1}(\psi(t(R \cdot)) \mathcal{F} f) \mid L_{p}\right\|^{q} \frac{d t}{t}\right)^{1 / q}
\end{aligned}
$$

and from here follows (13) for $R \geq 1, c_{1}, c_{2}, c_{3}>0$ and $s>\sigma_{p}$. 


\subsection{Proof of Theorem 2.8}

Proof. Step 1. At first we prove the left-hand side of (17). By (15) we have

$$
f_{j}^{a}\left(2^{-(j+1) a} x\right)=\sum_{k \in \mathbb{Z}^{n}} c_{k} f\left(x-2^{a} k\right), \quad c_{k} \in \mathbb{C}, \quad j \in \mathbb{N},
$$

where $f \in B_{p}^{s, a}$ and (14) is true. We would like to show that

$$
\left\|\sum_{k \in \mathbb{Z}^{n}} c_{k} f\left(\cdot-2^{a} k\right)\left|B_{p}^{s, a}\left\|\sim\left(\sum_{k \in \mathbb{Z}^{n}}\left|c_{k}\right|^{p}\right)^{1 / p}\right\| f\right| B_{p}^{s, a}\right\| .
$$

We use the characterization of $B_{p}^{s, a}$ via local means; see [7, 4.4]. Recall notation (3). Let $k \in C^{\infty}$ so that $\operatorname{supp} k \subset B^{a}=\left\{y \in \mathbb{R}^{n}:|y|_{a} \leq 1\right\}$ and

$$
k(t, f)(x)=\int_{\mathbb{R}^{n}} k(y) f\left(x+t^{a} y\right) d y, \quad t>0 .
$$

Let $k_{0} \in C^{\infty}$ such that $\operatorname{supp} k_{0} \subset B^{a}$, and $s_{1}>\max \left(s, \sigma_{p}\right)+\sigma_{p}$. Then

$$
\left\|f\left|B_{p}^{s, a}\|\sim\| k_{0}(1, f)\right| L_{p}\right\|+\left(\sum_{j=1}^{\infty} 2^{j s p}\left\|k\left(2^{-j}, f\right) \mid L_{p}\right\|^{p}\right)^{1 / p}
$$

is an equivalent quasi-norm in $B_{p}^{s, a}$; see $[7,4.4]$. We insert $(35)$ in (37) and obtain

$$
\begin{aligned}
k\left(t, \sum_{m \in \mathbb{Z}^{n}} c_{m} f\left(\cdot-2^{a} m\right)\right)(x) & =\int_{\mathbb{R}^{n}} k(y)\left(\sum_{m \in \mathbb{Z}^{n}} c_{m} f\left(x+t^{a} y-2^{a} m\right)\right) d y \\
& =\sum_{m \in \mathbb{Z}^{n}} c_{m} \int_{\mathbb{R}^{n}} k(y) f\left(x-2^{a} m+t^{a} y\right) m d y \\
& =\sum_{m \in \mathbb{Z}^{n}} c_{m} k(t, f)\left(x-2^{a} m\right)
\end{aligned}
$$

and it follows

$$
\begin{aligned}
\| \sum_{m \in \mathbb{Z}^{n}} c_{m} f(\cdot & \left.-2^{a} m\right) \mid B_{p}^{s, a} \| \\
\sim & \left\|k_{0}\left(1, \sum_{m \in \mathbb{Z}^{n}} c_{m} f\left(\cdot-2^{a} m\right)\right) \mid L_{p}\right\| \\
& +\left(\sum_{j=1}^{\infty} 2^{j s p}\left\|k\left(2^{-j}, \sum_{m \in \mathbb{Z}^{n}} c_{m} f\left(\cdot-2^{a} m\right)\right) \mid L_{p}\right\|^{p}\right)^{\frac{1}{p}} \\
& \sim\left(\sum_{m \in \mathbb{Z}^{n}}\left|c_{m}\right|^{p}\right)^{1 / p}\left(\left\|k_{0}(1, f) \mid L_{p}\right\|+\left(\sum_{j=1}^{\infty} 2^{j s p}\left\|k\left(2^{-j}, f\right) \mid L_{p}\right\|^{p}\right)^{\frac{1}{p}}\right) \\
& \sim\left(\sum_{m \in \mathbb{Z}^{n}}\left|c_{m}\right|^{p}\right)^{1 / p}\left\|f \mid B_{p}^{s, a}\right\| .
\end{aligned}
$$


Now the left-hand side of inequality of (17) is an easy consequence of Proposition 2.7, (35) and (36):

$$
\begin{aligned}
\left\|f_{j}^{a} \mid B_{p}^{s, a}\right\| & \leq c 2^{j(s-n / p)}\left\|\sum_{m \in \mathbb{Z}^{n}} c_{m} f\left(\cdot-2^{a} m\right) \mid B_{p}^{s, a}\right\| \\
& \leq c^{\prime} 2^{j(s-n / p)}\left(\sum_{m \in \mathbb{Z}^{n}}\left|c_{m}\right|^{p}\right)^{1 / p}\left\|f \mid B_{p}^{s, a}\right\| .
\end{aligned}
$$

Step 2. In this step we prove the right-hand side of (17). For this we would like to use the localization property given in $[4,2.3 .2]$ if $n=1$ and for the functions

$$
f^{j \alpha}(x)=\sum_{m \in \mathbb{Z}} c_{m} f\left(2^{(j+1) \alpha} x-2^{\alpha} m\right), \quad c_{m} \in \mathbb{C}, \quad j, \alpha \in \mathbb{N}
$$

where $f \in \mathcal{S}^{\prime}(\mathbb{R})$. By $[4,2.3 .2 / 4]$ we know that there exist two constants $c^{\prime}>0$ and $c^{\prime \prime}>0$ such that for all $f \in B_{p p}^{s}$

$$
c^{\prime}\left\|f^{j \alpha}\left|B_{p p}^{s}\left\|\leq 2^{j \alpha\left(s-\frac{1}{p}\right)}\left(\sum_{k \in \mathbb{Z}}\left|c_{k}\right|^{p}\right)^{1 / p}\right\| f\right| B_{p p}^{s}\right\| \leq c^{\prime \prime}\left\|f^{j \alpha} \mid B_{p p}^{s}\right\|,
$$

as for $n=1$ isotropic and anisotropic results coincide. For the functions $f_{j}^{a}$ given in (15) we use the Fubini property of $B_{p}^{s, a}$; see $[2,6$.$] , i.e.,$

$$
\begin{aligned}
& \left\|f_{j}^{a} \mid B_{p}^{s, a}\left(\mathbb{R}^{n}\right)\right\| \\
& \quad \sim \sum_{m=1}^{n}\|\| f_{j}^{a}\left(x_{1}, \ldots, x_{m-1}, \cdot, x_{m+1}, \ldots, x_{n}\right)\left|B_{p}^{s_{m}}(\mathbb{R})\left\|_{x_{m}} \mid L_{p}\left(\mathbb{R}^{n-1}\right)\right\|_{x^{\prime}},\right.
\end{aligned}
$$

where $x^{\prime}=\left(x_{1}, \ldots, x_{m-1}, x_{m+1}, \ldots, x_{n}\right)$ and $s_{m}=\frac{s}{a_{m}}$. By (15) and (16)

$$
\begin{aligned}
\left\|f_{j}^{a}\left(x_{1}, \ldots, x_{m-1}, \cdot, x_{m+1}, \ldots, x_{n}\right) \mid B_{p}^{s_{m}}(\mathbb{R})\right\|_{x_{m}} \\
=\left\|\sum_{k_{m}=-\infty}^{\infty} f_{m}\left(2^{(j+1) a_{m}} x_{m}-2^{a_{m}} k_{m}\right)\left[\sum_{\bar{k} \in \mathbb{Z}^{n-1}} c_{\left(k_{1}, \ldots, k_{n}\right)} \bar{f}\right] \mid B_{p}^{s_{m}}(\mathbb{R})\right\|_{x_{m}},
\end{aligned}
$$

where $\bar{k}=\left(k_{1}, \ldots, k_{m-1}, k_{m+1}, \ldots, k_{n}\right)$ and

$$
\begin{aligned}
\bar{f}=f_{1}\left(2^{(j+1) a_{1}}\right. & \left.x_{1}-2^{a_{1}} k_{1}\right) \cdots f_{m-1}\left(2^{(j+1) a_{m-1}} x_{m-1}-2^{a_{m-1}} k_{m-1}\right) \\
& \times f_{m+1}\left(2^{(j+1) a_{m+1}} x_{m+1}-2^{a_{m+1}} k_{m+1}\right) \cdots f_{n}\left(2^{(j+1) a_{n}} x_{1}-2^{a_{n}} k_{n}\right) .
\end{aligned}
$$

Let $d_{k_{m}}=\left(\sum_{\substack{l \in \mathbb{Z}^{n} \\ l_{m}=k_{m}}}\left|c_{l}\right|^{p}\right)^{1 / p}$ and without restriction of generality we may assume 
that $d_{k_{m}}>0$. We have that

$$
\begin{aligned}
& \left\|f_{j}^{a}\left(x_{1}, \ldots, x_{m-1}, \cdot, x_{m+1}, \ldots, x_{n}\right) \mid B_{p}^{s_{m}}(\mathbb{R})\right\|_{x_{m}} \\
& \quad=\left\|\sum_{k_{m}=-\infty}^{\infty} f_{m}\left(2^{(j+1) a_{m}} x_{m}-2^{a_{m}} k_{m}\right)\left[\sum_{\bar{k} \in \mathbb{Z}^{n-1}} d_{k_{m}} \frac{c_{\left(k_{1}, \ldots, k_{n}\right)}}{d_{k_{m}}}\right] \mid B_{p}^{s_{m}}(\mathbb{R})\right\|_{x_{m}} .
\end{aligned}
$$

Let $\overline{c_{k}}=\frac{c_{\left(k_{1}, \ldots, k_{n}\right)}}{d_{k_{m}}}$ and by $(40)$ we get that

$$
\begin{aligned}
\left\|f_{j}^{a}\left(x_{1}, \ldots, x_{m-1}, \cdot, x_{m+1}, \ldots, x_{n}\right) \mid B_{p}^{s_{m}}(\mathbb{R})\right\|_{x_{m}} \\
\quad=\left\|\sum_{k_{m}=-\infty}^{\infty} d_{k_{m}} f_{m}\left(2^{(j+1) a_{m}} x_{m}-2^{a_{m}} k_{m}\right)\left[\sum_{\bar{k} \in \mathbb{Z}^{n-1}} \overline{c_{k}} \bar{f}\right] \mid B_{p}^{s_{m}}(\mathbb{R})\right\|_{x_{m}} \\
\quad=\left[\sum_{\bar{k} \in \mathbb{Z}^{n-1}} \overline{c_{k}} \bar{f}\right]\left\|\sum_{k_{m}=-\infty}^{\infty} d_{k_{m}} f_{m}\left(2^{(j+1) a_{m}} x_{m}-2^{a_{m}} k_{m}\right) \mid B_{p}^{s_{m}}(\mathbb{R})\right\|_{x_{m}} .
\end{aligned}
$$

By (41),

$$
\begin{aligned}
& \|\| f_{j}^{a}\left(x_{1}, \ldots, x_{m-1}, \cdot, x_{m+1}, \ldots, x_{n}\right)\left|B_{p}^{s_{m}}(\mathbb{R})\left\|_{x_{m}} \mid L_{p}\left(\mathbb{R}^{n-1}\right)\right\|_{x^{\prime}}\right. \\
& \left.=\| \sum_{\bar{k} \in \mathbb{Z}^{n-1}} \overline{c_{k}} \bar{f}\right]\left\|_ { k _ { m } = - \infty } ^ { \infty } d _ { k _ { m } } f _ { m } ( 2 ^ { ( j + 1 ) a _ { m } } x _ { m } - 2 ^ { a _ { m } } k _ { m } ) \left|B_{p}^{s_{m}}(\mathbb{R})\left\|_{x_{m}} \mid L_{p}\left(\mathbb{R}^{n-1}\right)\right\|_{x^{\prime}}\right.\right. \\
& =\left\|\sum_{k_{m}=-\infty}^{\infty} d_{k_{m}} f_{m}\left(2^{(j+1) a_{m}} x_{m}-2^{a_{m}} k_{m}\right) \mid B_{p}^{s_{m}}(\mathbb{R})\right\|_{x_{m}} \\
& \quad \times\left\|\sum_{\bar{k} \in \mathbb{Z}^{n-1}} \overline{c_{k}} \bar{f} \mid L_{p}\left(\mathbb{R}^{n-1}\right)\right\|_{x^{\prime}} .
\end{aligned}
$$

Note that

$$
\begin{aligned}
\left\|\sum_{\bar{k} \in \mathbb{Z}^{n-1}} \overline{c_{k}} \bar{f} \mid L_{p}\left(\mathbb{R}^{n-1}\right)\right\|_{x^{\prime}}=\left(\sum_{\bar{k} \in \mathbb{Z}^{n-1}}\left|\overline{c_{k}}\right|^{p}\right)^{1 / p} 2^{(j+1)\left(a_{m}-n\right) / p} & \\
& \times\left\|f_{1} \cdots f_{m-1} f_{m+1} \cdots f_{n} \mid L_{p}\left(\mathbb{R}^{n-1}\right)\right\|_{x^{\prime}} .
\end{aligned}
$$

(Recall that $-\left(a_{1}+\cdots+a_{m-1}+a_{m+1}+\cdots+a_{n}\right)=a_{m}-n$.) Now we use (38) for the spaces $B_{p}^{s_{m}}(\mathbb{R})$ and by $(42),(43)$

$$
\begin{aligned}
\|\| f_{j}^{a}\left(x_{1}, \ldots, x_{m-1}, \cdot, x_{m+1}, \ldots, x_{n}\right)\left|B_{p}^{s_{m}}(\mathbb{R})\left\|_{x_{m}} \mid L_{p}\left(\mathbb{R}^{n-1}\right)\right\|_{x^{\prime}}\right. \\
\geq c^{\prime} 2^{j a_{m}\left(s_{m}-\frac{1}{p}\right)}\left(\sum_{k_{m}=-\infty}^{\infty}\left|d_{k_{m}}\right|^{p}\right)^{1 / p}\left\|f_{m} \mid B_{p}^{s_{m}}(\mathbb{R})\right\|_{x_{m}} \\
\quad \times 2^{\frac{j}{p}\left(a_{m}-n\right)}\left(\sum_{\bar{k} \in \mathbb{Z}^{n-1}}\left|\overline{c_{k}}\right|^{p}\right)^{\frac{1}{p}}\left\|f_{1} \cdots f_{m-1} \cdot f_{m+1} \cdots f_{n} \mid L_{p}\left(\mathbb{R}^{n-1}\right)\right\|_{x^{\prime}} .
\end{aligned}
$$


On the other hand,

$$
\left(\sum_{k_{m}=-\infty}^{\infty}\left|d_{k_{m}}\right|^{p}\right)^{1 / p}=\left(\sum_{k_{m}=-\infty}^{\infty} \sum_{\substack{l \in \mathbb{Z}^{n} \\ l_{m}=k_{m}}}\left|c_{l}\right|^{p}\right)^{1 / p}=\left(\sum_{l \in \mathbb{Z}^{n}}^{\infty}\left|c_{l}\right|^{p}\right)^{1 / p}
$$

and

$$
\begin{aligned}
\left(\sum_{\bar{k} \in \mathbb{Z}^{n-1}}\left|\overline{c_{k}}\right|^{p}\right)^{1 / p} & =\left(\sum_{\bar{k} \in \mathbb{Z}^{n-1}} \frac{\left|c_{\left(k_{1}, \ldots, k_{n}\right)}\right|^{p}}{d_{k_{m}}^{p}}\right)^{1 / p} \\
& =\frac{1}{d_{k_{m}}} \underbrace{\left(\sum_{\bar{k} \in \mathbb{Z}^{n-1}}\left|c_{\left(k_{1}, \ldots, k_{n}\right)}\right|^{p}\right)^{1 / p} \geq 1 .}_{\geq d_{k_{m}}} .
\end{aligned}
$$

By (44), (45) and (46) and $s_{m} \cdot a_{m}=s$, we conclude

$$
\begin{aligned}
\|\| f_{j}^{a}\left(x_{1}, \ldots,\right. & \left.x_{m-1}, \cdot, x_{m+1}, \ldots, x_{n}\right)\left|B_{p}^{s_{m}}(\mathbb{R})\left\|_{x_{m}} \mid L_{p}\left(\mathbb{R}^{n-1}\right)\right\|_{x^{\prime}}\right. \\
\geq & c^{\prime} 2^{j\left(s-\frac{n}{p}\right)}\left(\sum_{k \in \mathbb{Z}^{n}}\left|c_{k}\right|^{p}\right)^{1 / p}\left\|f_{m} \mid B_{p}^{s_{m}}(\mathbb{R})\right\|_{x_{m}} \\
& \times\left\|f_{1} \cdots f_{m-1} \cdot f_{m+1} \cdots f_{n} \mid L_{p}\left(\mathbb{R}^{n-1}\right)\right\|_{x^{\prime}} \\
\geq & c^{\prime} 2^{j\left(s-\frac{n}{p}\right)}\left(\sum_{k \in \mathbb{Z}^{n}}\left|c_{k}\right|^{p}\right)^{1 / p}\|\| f_{1} \cdots f_{n}\left|B_{p}^{s_{m}}(\mathbb{R})\left\|_{x_{m}} \mid L_{p}\left(\mathbb{R}^{n-1}\right)\right\|_{x^{\prime}} .\right.
\end{aligned}
$$

By (47) and the Fubini property (39) we obtain the right-hand side of inequality of (17)

$$
\begin{aligned}
\left\|f_{j}^{a} \mid B_{p}^{s, a}\right\| & \geq c 2^{j\left(s-\frac{n}{p}\right)}\left(\sum_{k \in \mathbb{Z}^{n}}\left|c_{k}\right|^{p}\right)^{1 / p} \sum_{m=1}^{n}\|\| f_{1} \cdots f_{n}\left|B_{p}^{s_{m}}(\mathbb{R})\left\|_{x_{m}} \mid L_{p}\left(\mathbb{R}^{n-1}\right)\right\|_{x^{\prime}}\right. \\
& \geq c^{\prime} 2^{j\left(s-\frac{n}{p}\right)}\left(\sum_{k \in \mathbb{Z}^{n}}\left|c_{k}\right|^{p}\right)^{1 / p}\left\|f \mid B_{p}^{s, a}\left(\mathbb{R}^{n}\right)\right\| .
\end{aligned}
$$

\subsection{Wavelet frames}

In the sequel we describe wavelet frames which are an effective instrument to estimate approximation numbers. This will be needed in our proofs below. Let $k$ be a nonnegative $C^{\infty}$ function in $\mathbb{R}^{n}$ with

$$
\operatorname{supp} k \subset\left\{y \in \mathbb{R}^{n}:|y|_{a}<2^{J}, y_{j}>0\right\},
$$

for some $J \in \mathbb{N}$, and

$$
\sum_{m \in \mathbb{Z}^{n}} k(x-m)=1, \quad x \in \mathbb{R}^{n}
$$


Recall that $x^{\beta}=x_{1}^{\beta_{1}} \cdots x_{n}^{\beta_{n}}$ where $x=\left(x_{1}, \ldots, x_{n}\right) \in \mathbb{R}^{n}$ and $\beta \in \mathbb{N}_{0}^{n}$, and put

$$
k^{\beta}(x)=\left(2^{-J a} x\right)^{\beta} k(x) \geq 0, \quad x \in \mathbb{R}^{n}, \quad \beta \in \mathbb{N}_{0}^{n} .
$$

Let

$$
\omega \in \mathcal{S}, \quad \operatorname{supp} \omega \subset(-\pi, \pi)^{n}, \quad \omega(x)=1 \quad \text { if } \quad|x|_{a} \leq 2,
$$

and let

$$
\omega^{\beta}(x)=\frac{i^{|\beta|} 2^{J a \beta}}{(2 \pi)^{n} \beta !} x^{\beta} \omega(x) \quad \text { for } \quad x \in \mathbb{R}^{n}, \quad \beta \in \mathbb{N}_{0}^{n},
$$

and

$$
\Omega^{\beta}(x)=\sum_{m \in \mathbb{Z}^{n}}\left(\omega^{\beta}\right)^{\vee}(m) e^{-i m x}, \quad x \in \mathbb{R}^{n}, \quad \beta \in \mathbb{N}_{0}^{n},
$$

where $|\beta|=\beta_{1}+\cdots+\beta_{n}$, and $\beta !=\beta_{1} ! \ldots \beta_{n}$ ! and $a \beta=a_{1} \beta_{1}+\cdots+a_{n} \beta_{n}$. Let $\varphi_{0}$ be a $C^{\infty}$ function in $\mathbb{R}^{n}$ with

$$
\varphi_{0}(x)=1 \quad \text { if } \quad|x|_{a} \leq 1 \quad \text { and } \quad \varphi_{0}(x)=0 \quad \text { if } \quad|x|_{a} \geq \frac{3}{2},
$$

and let $\varphi(x)=\varphi_{0}(x)-\varphi_{0}\left(2^{a} x\right)$. Then

$$
\Phi_{j m}^{\beta}(x)= \begin{cases}\Phi_{F}^{\beta}(x-m), & \text { if } j=0 \\ \Phi_{M}^{\beta}\left(2^{j a} x-m\right), & \text { if } j \in \mathbb{N}\end{cases}
$$

are analytic wavelets where the father wavelets $\Phi_{F}^{\beta}(x)$ and the mother wavelets $\Phi_{M}^{\beta}(x)$ are given by their inverse Fourier transforms

$$
\begin{array}{ll}
\left(\Phi_{F}^{\beta}\right)^{\vee}(\xi)=\varphi_{0}(\xi) \Omega^{\beta}(\xi), & \xi \in \mathbb{R}^{n}, \\
\left(\Phi_{M}^{\beta}\right)^{\vee}(\xi)=\varphi(\xi) \Omega^{\beta}(\xi), & \xi \in \mathbb{R}^{n} .
\end{array}
$$

For the sequence

$$
\lambda=\left\{\lambda_{j m}^{\beta} \in \mathbb{C}: j \in \mathbb{N}_{0}, m \in \mathbb{Z}^{n}, \beta \in \mathbb{N}_{0}^{n}\right\},
$$

$s \in \mathbb{R}, 0<p \leq \infty$, and $\varrho \geq 0$, we put

$$
\left\|\lambda \mid b_{p}^{s, \varrho}\right\|=\left(\sum_{\beta \in \mathbb{N}_{0}^{n}} \sum_{j=0}^{\infty} \sum_{m \in \mathbb{Z}^{n}} 2^{\varrho a \beta p+j(s-n / p) p}\left|\lambda_{j m}^{\beta}\right|^{p}\right)^{1 / p} .
$$

For $f \in L_{p}\left(\mathbb{R}^{n}\right)$,

$$
\lambda_{j m}^{\beta}(f)=2^{j n} \int_{\mathbb{R}^{n}} f(x) \Phi_{j m}^{\beta}(x) d x, \quad j \in \mathbb{N}_{0}, \quad m \in \mathbb{Z}^{n}, \quad \beta \in \mathbb{N}_{0}^{n} .
$$

We make use of the following wavelet characterization. 
Theorem 4.1 ([9, Theorem 2.1]). Let $0<p<\infty, s>\sigma_{p}$ where $\sigma_{p}=n\left(\frac{1}{p}-1\right)_{+}$, $\varrho \geq 0$, and a an anisotropy. Then $f \in \mathcal{S}^{\prime}$ is an element of $B_{p}^{s, a}$ if, and only if, it can be represented as

$$
f=\sum_{\beta \in \mathbb{N}_{0}^{n}} \sum_{j=0}^{\infty} \sum_{m \in \mathbb{Z}^{n}} \lambda_{j m}^{\beta} k^{\beta}\left(2^{j a} x-m\right), \quad x \in \mathbb{R}^{n},
$$

with $\left\|\lambda \mid b_{p}^{s, \varrho}\right\|<\infty$, absolute convergence being in $L_{\max (1, p)}$. Furthermore,

$$
\left\|f\left|B_{p}^{s, a}\|\sim \inf \| \lambda\right| b_{p}^{s, \varrho}\right\|
$$

where the infimum is taken over all admissible representations (50). In addition, any $f \in B_{p}^{s, a}$ can be optimally represented by

$$
f=\sum_{\beta \in \mathbb{N}_{0}^{n}} \sum_{j=0}^{\infty} \sum_{m \in \mathbb{Z}^{n}} \lambda_{j m}^{\beta}(f) k^{\beta}\left(2^{j a} x-m\right),
$$

with

$$
\left\|f\left|B_{p}^{s, a}\|\sim\| \lambda(f)\right| b_{p}^{s, \varrho}\right\| .
$$

Remark 4.2. In the sequel we shall stick to the notation

$$
k_{j m}^{\beta}(x)=k^{\beta}\left(2^{j a} x-m\right), \quad \beta \in \mathbb{N}_{0}^{n}, \quad j \in \mathbb{N}_{0}, \quad m \in \mathbb{Z}^{n} .
$$

\subsection{Proof of Proposition 3.1}

We closely follow the ideas in [21].

Proof. Step 1. We prove the existence of (22) like in the isotropic case, see [20, Theorem 9.3, Corollary 9.8; 21, Prop. 2]. In our case we use the anisotropic local means and the equivalent norm in anisotropic function spaces, see [8, 2.2]. In comparison with [20, Theorem 9.3] we need only a special case where $u=v=p^{\prime}$ and $\sigma=-s$. On the other hand, the existence of $\operatorname{tr}_{\Gamma}$ can also be shown by similar arguments as presented below.

Step 2. Let $f \in B_{p}^{s, a}$ be given by (51), (52). (We use the notation (53).) For any fixed $\beta \in \mathbb{N}_{0}^{n}$ we have

$$
\begin{aligned}
\left\|\sum_{j=0}^{\infty} \sum_{m \in \mathbb{Z}^{n}} \lambda_{j m}^{\beta}(f) k_{j m}^{\beta} \mid L_{p}(\Gamma)\right\| & \leq \sum_{j=0}^{\infty}\left\|\sum_{m \in \mathbb{Z}^{n}} \lambda_{j m}^{\beta}(f) k_{j m}^{\beta} \mid L_{p}(\Gamma)\right\| \\
& \leq c \sum_{j=0}^{\infty}\left(\int_{\mathbb{R}^{n}} \sum_{m \in \mathbb{Z}^{n}}\left|\lambda_{j m}^{\beta}(f)\right|^{p}\left|k_{j m}^{\beta}(x)\right|^{p} \mu(d x)\right)^{1 / p}
\end{aligned}
$$




$$
\begin{aligned}
& \leq c \sum_{j=0}^{\infty}\left(\sum_{m \in \mathbb{Z}^{n}}\left|\lambda_{j m}^{\beta}(f)\right|^{p} \int_{c Q_{j m}^{a}}\left|k_{j m}^{\beta}(x)\right|^{p} \mu(d x)\right)^{1 / p} \\
& \leq c^{\prime} \sum_{j=0}^{\infty} \mu_{j}^{\frac{1}{p}}\left(\sum_{m \in \mathbb{Z}^{n}}\left|\lambda_{j m}^{\beta}(f)\right|^{p}\right)^{1 / p}
\end{aligned}
$$

where we used the boundedness of $k$ and (19). We apply the Hölder inequality, recall that $\frac{1}{p}+\frac{1}{p^{\prime}}=1$, and so we can continue

$$
\begin{aligned}
\left\|\sum_{j=0}^{\infty} \sum_{m \in \mathbb{Z}^{n}} \lambda_{j m}^{\beta}(f) k_{j m}^{\beta} \mid L_{p}(\Gamma)\right\| & \\
\leq & c^{\prime}\left(\sum_{j=0}^{\infty} 2^{-j p^{\prime}\left(s-\frac{n}{p}\right)} \mu_{j}^{\frac{p^{\prime}}{p}}\right)^{1 / p^{\prime}}\left(\sum_{j, m} 2^{j\left(s-\frac{n}{p}\right) p}\left|\lambda_{j m}^{\beta}(f)\right|^{p}\right)^{1 / p} .
\end{aligned}
$$

We choose $\varrho>0$. Then it follows by (49) and (52) that

$$
\left\|\operatorname{tr}_{\Gamma} f\left|L_{p}(\Gamma)\left\|\leq c^{\prime}\left(\sum_{j=0}^{\infty} 2^{-j p^{\prime}\left(s-\frac{n}{p}\right)} \mu_{j}^{p^{\prime}-1}\right)^{1 / p^{\prime}}\right\| f\right| B_{p}^{s, a}\right\|
$$

where $c^{\prime}$ is independent of $\mu$. This proves (23).

Step 3. We prove that $\operatorname{tr}_{\Gamma}$ is compact. Let $B \in \mathbb{N}, J \in \mathbb{N},[a \beta]=\max \{r \in \mathbb{Z}$ : $r \leq a \beta\}$, and let $\operatorname{tr}_{\Gamma}^{B, J}$ be given by

$$
\operatorname{tr}_{\Gamma}^{B, J} f=\sum_{[a \beta] \leq B} \sum_{j \leq J} \sum_{m \in \mathbb{Z}^{n}}^{\Gamma} \lambda_{j m}^{\beta}(f) k_{j m}^{\beta},
$$

where again $f \in B_{p}^{s, a}$ is given by (51),(52) and where the sum $\sum_{m \in \mathbb{Z}^{n}}^{\Gamma}$ is restricted to those $m \in \mathbb{Z}^{n}$ such that the rectangles $Q_{j m}^{a}$ have a non-empty intersection with $\Gamma$. For given $\delta>0$ and suitably chosen $\varrho>0$ it follows by the above arguments for $f \in B_{p}^{s, a}$ having norm of at most 1 that

$$
\begin{aligned}
\left\|\left(\operatorname{tr}_{\Gamma}-\operatorname{tr}_{\Gamma}^{B, J}\right) f \mid L_{p}(\Gamma)\right\| \\
\leq c\left(\sum_{[a \beta] \geq B} 2^{-\delta a \beta}\right)+c\left(\sum_{[a \beta] \leq B} 2^{-\delta a \beta}\right)\left(\sum_{j \geq J} 2^{-j p^{\prime}\left(s-\frac{n}{p}\right)} \mu_{j}^{p^{\prime}-1}\right)^{1 / p^{\prime}},
\end{aligned}
$$

see (49) and (54), (55). By (21) we find for any given $\varepsilon>0$ sufficiently large numbers $B$ and $J$ such that

$$
\left\|\operatorname{tr}_{\Gamma}-\operatorname{tr}_{\Gamma}^{B, J}\right\| \leq \varepsilon
$$

Then $\operatorname{tr}_{\Gamma}$ is compact, as $\operatorname{tr}_{\Gamma}^{B, J}$ are finite rank operators. 


\subsection{Proof of Proposition 3.3}

Proof. Note that (25) implies (21), thus by Proposition 3.1 the operator $\operatorname{tr}_{\Gamma}$ is compact. We refine (56) by

$$
\operatorname{tr}_{\Gamma}^{J} f=\sum_{[a \beta] \leq J} \sum_{j \leq J-[a \beta]} \sum_{m \in \mathbb{Z}^{n}}^{\Gamma} \lambda_{j m}^{\beta}(f) k_{j m}^{\beta}, \quad J \in \mathbb{N},
$$

where again $f \in B_{p}^{s, a}$ is given by (51), (52) and the last sum has the same meaning as the last sum in (56). As $\mu$ is a measure in $\mathbb{R}^{n}$ we have that

$$
\mu_{K} \leq c 2^{(J-K) n} \mu_{J}, \quad K \leq J
$$

also in the anisotropic case. (Recall that $a_{1}+\cdots+a_{n}=n$.) Let $\delta>0$ be sufficiently large. By (59) we obtain for $f \in B_{p}^{s, a}$ having norm of at most 1 in analogy to (57) that

$$
\begin{aligned}
\left\|\left(\operatorname{tr}_{\Gamma}-\operatorname{tr}_{\Gamma}^{J}\right) f \mid L_{p}(\Gamma)\right\| & \leq c 2^{-\delta J}+c \sum_{[a \beta] \leq J} 2^{-\delta a \beta}\left(\sum_{j \geq J-[a \beta]} 2^{-j p^{\prime}\left(s-\frac{n}{p}\right)} \mu_{j}^{\frac{p^{\prime}}{p}}\right)^{1 / p^{\prime}} \\
& \leq c 2^{-\delta J}+c \sum_{[a \beta] \leq J} 2^{-\delta a \beta} 2^{-(J-[a \beta])\left(s-\frac{n}{p}\right)} \mu_{J-[a \beta]}^{\frac{1}{p}} \\
& \leq c 2^{-\delta J}+c \mu_{J}^{\frac{1}{p}} 2^{-J\left(s-\frac{n}{p}\right)} \sum_{[a \beta] \leq J} 2^{-\delta a \beta+a \beta\left(s-\frac{n}{p}\right)+a \beta \frac{n}{p}} \\
& \leq c^{\prime} 2^{-J\left(s-\frac{n}{p}\right)} \mu_{J}^{\frac{1}{p}} .
\end{aligned}
$$

In the second estimate we used assumption (25) and in the next one (59). For the rank of $\operatorname{tr}_{\Gamma}^{J}$ we have the estimate

$$
\operatorname{rank}\left(\operatorname{tr}_{\Gamma}^{J}\right) \leq c \sum_{[a \beta] \leq J} 2^{n(J-[a \beta])} \leq c^{\prime} 2^{n J}
$$

This proves (26).

\subsection{Proof of Proposition 3.5}

Proof. For simplicity we prove this proposition for the case $n=2$. If $n>2$ this can be done in a similar way.

We use the well-known mass distribution procedure to construct a measure $\mu$ with the desired properties. We refer to $[19$, ch. 4$]$ for details. Let $Q=[0,1]^{2}$ be the closed cube with side-length 1 , we take the affine contractions $\left(A_{m}\right)_{m=1}^{N}$ on $\mathbb{R}^{2}$ which map the unit square to the rectangles $\left(A_{m} Q\right)_{m=1}^{N}$ with side-lengths $r^{a_{1}}$ and $r^{a_{2}}$ where $0<a_{1}<a_{2}$ and $a_{1}+a_{2}=2$ as in figure 1 , so that they are disjoint and 


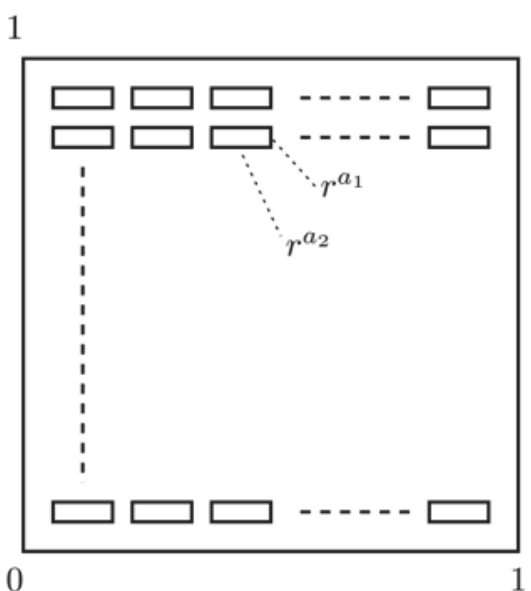

Figure 1

$\mu\left(A_{m} Q\right)=N^{-1}$. Furthermore we have $N r^{2}=N\left|A_{m} Q\right|<1$. Let

$$
\begin{gathered}
A Q=(A Q)^{1}=\bigcup_{m=1}^{N} A_{m} Q, \quad(A Q)^{0}=Q, \\
(A Q)^{k}=A\left((A Q)^{k-1}\right) .
\end{gathered}
$$

The sequence of sets is monotonically decreasing and by [6, Theorem 8.3]

$$
\Gamma=(A Q)^{\infty}=\bigcap_{k \in \mathbb{N}}(A Q)^{k}=\lim _{k \rightarrow \infty}(A Q)^{k}
$$

is the uniquely determined fractal generated by the contractions $\left(A_{m}\right)_{m=1}^{N}$. But on the other hand we assume that $\mu\left(A_{m} Q\right)=r^{d}$, where $m=1, \ldots, N$, and from here we get that $d=\frac{\log N}{|\log r|}$. If $0<d<2$ then it follows by elementary geometrical reasoning that one can finds (sufficiently small) numbers $r>0$ and suitably chosen natural numbers $N \in \mathbb{N}$ with the desired properties.

\subsection{Proof of Theorem 3.7}

Proof. Step 1. First we prove the right-hand side of the estimate (28) in Theorem 3.7. Again we use the wavelet expansion (51), (52). For fixed $\beta \in \mathbb{N}_{0}^{n}$ we put

$$
\operatorname{tr}_{\Gamma}^{\beta} f=\sum_{j \in \mathbb{N}_{0}} \sum_{m \in \mathbb{Z}^{n}}^{\Gamma} \lambda_{j m}^{\beta}(f) k_{j m}^{\beta}
$$


and

$$
\operatorname{tr}_{\Gamma}^{\beta, J} f=\sum_{j \leq J} \sum_{m \in \mathbb{Z}^{n}}^{\Gamma} \lambda_{j m}^{\beta}(f) k_{j m}^{\beta},
$$

where the second sum has the same meaning as the last sum in (56). By the same reasoning as in (58) and (60) but now for fixed $\beta$ we have for $f \in B_{p}^{s, a}$ with norm of at most 1 ,

$$
\left\|\left(\operatorname{tr}_{\Gamma}^{\beta}-\operatorname{tr}_{\Gamma}^{\beta, J}\right) f \mid L_{p}(\Gamma)\right\| \leq c 2^{-\delta a \beta} 2^{J\left(\frac{n}{p}-s\right)} \mu_{J}^{\frac{1}{p}} .
$$

By Definition 3.4 there exists a constant $c>0$ independent of $j \in \mathbb{N}_{0}$ with $\mu\left(Q_{j m}^{a} \cap \Gamma\right)$ $\leq c 2^{-j d}$ and we obtain that

$$
\left\|\left(\operatorname{tr}_{\Gamma}^{\beta}-\operatorname{tr}_{\Gamma}^{\beta, J}\right) f \mid L_{p}(\Gamma)\right\| \leq c 2^{-\delta a \beta} 2^{J\left(\frac{n}{p}-s\right)} 2^{-J \frac{d}{p}} .
$$

In definition (24) put $L=\operatorname{tr}_{\Gamma}^{\beta, J}, T=\operatorname{tr}_{\Gamma}^{\beta}$, and note that for $j \in \mathbb{N}_{0}$,

$$
\operatorname{rank}\left(\sum_{m \in \mathbb{Z}^{n}}^{\Gamma} \lambda_{j m}^{\beta}(f) k_{j m}^{\beta}\right) \leq c 2^{j d} .
$$

Thus we obtain by (61) that

$$
\operatorname{rank}\left(\operatorname{tr}_{\Gamma}^{\beta, J}\right) \leq c \sum_{j \leq J} 2^{j d} \leq c^{\prime} 2^{J d} .
$$

Then (62) implies that there are two positive numbers $c$ and $c^{\prime}$ such that

$$
a_{c 2^{J d}}\left(\operatorname{tr}_{\Gamma}^{\beta}\right) \leq c^{\prime} 2^{-\delta a \beta} 2^{J\left(\frac{n}{p}-s\right)} 2^{-J \frac{d}{p}} .
$$

For $k \in \mathbb{N}$ there are numbers $J_{k} \in \mathbb{N}$ such that

$$
2^{J_{k} d} \sim k \quad \text { with } \quad J_{1} \leq J_{2} \leq \cdots \leq J_{n} \leq \cdots ;
$$

inserted in (63) this leads to

$$
a_{c k}\left(\operatorname{tr}_{\Gamma}^{\beta}\right) \leq c 2^{-\delta a \beta} 2^{J_{k}\left(\frac{n}{p}-s\right)} k^{-\frac{1}{p}} .
$$

Let $\varepsilon>0$, for given $k \in \mathbb{N}$ we apply $(65)$ to $k_{\beta} \in \mathbb{N}$ with $k_{\beta} \sim 2^{-\varepsilon a \beta} k$. Then it follows by the additivity property of approximation numbers and from (65) that

$$
\begin{aligned}
a_{c k}\left(\operatorname{tr}_{\Gamma}^{\beta}\right) & \leq \sum_{\beta \in \mathbb{N}_{0}^{n}} a_{k_{\beta}}\left(\operatorname{tr}_{\Gamma}^{\beta}\right) \\
& \leq c^{\prime} \sum_{\beta \in \mathbb{N}_{0}^{n}} 2^{-\delta a \beta} 2^{J_{k_{\beta}}\left(\frac{n}{p}-s\right)}\left(2^{-\varepsilon a \beta} k\right)^{-\frac{1}{p}} \\
& \leq c^{\prime \prime} 2^{J_{k}\left(\frac{n}{p}-s\right)} k^{-\frac{1}{p}} \sum_{\beta \in \mathbb{N}_{0}^{n}} 2^{-a \beta\left(\delta-\frac{\varepsilon}{p}\right)} \\
& \leq c^{\prime \prime \prime} 2^{J_{k}\left(\frac{n}{p}-s\right)} k^{-\frac{1}{p}}
\end{aligned}
$$


for $\varepsilon>0$ small. We used $s \leq \frac{n}{p}$, such that $J_{k_{\beta}}\left(\frac{n}{p}-s\right) \leq J_{k}\left(\frac{n}{p}-s\right)$. Finally (64) implies

$$
a_{c k}\left(\operatorname{tr}_{\Gamma}^{\beta}\right) \leq c^{\prime \prime \prime} k^{\frac{1}{d}\left(\frac{n}{p}-s\right)-\frac{1}{p}}
$$

and so we finished the proof of the right-hand side of the estimate (28).

Step 2. To verify the left-hand side of the estimate (28) we closely follow the argument in $[21,4.4]$ for the isotropic case. Let $J \in \mathbb{N}$ and $c>0$ be suitably chosen numbers such that there are lattice points

$$
\gamma_{j, l}=2^{(-j-J) a} m \quad \text { with } \quad m \in \mathbb{Z}^{n}, \quad l=1, \ldots, M_{j} \quad \text { where } \quad M_{j} \sim 2^{j d}
$$

with

$$
\operatorname{dist}\left(\gamma_{j, l}, \Gamma\right) \leq c 2^{-j} \quad \text { and disjoint anisotropic balls } \quad B^{a}\left(\gamma_{j, l}, c 2^{-j+1}\right) .
$$

With $k$ as in (48) we put for $j \in \mathbb{N}_{0}$,

$$
f_{j}^{a}(x)=\sum_{l=1}^{M_{j}} c_{j l} 2^{-j\left(s-\frac{n}{p}\right)} k\left(2^{j a}\left(x-\gamma_{j, l}\right)\right), \quad c_{j l} \in \mathbb{C}, \quad x \in \mathbb{R}^{n} .
$$

Then we obtain by Theorem 2.8

$$
\left\|f_{j}^{a} \mid B_{p}^{s, a}\right\| \sim 2^{j\left(s-\frac{n}{p}\right)}\left(\sum_{l=1}^{M_{j}} 2^{-j\left(s-\frac{n}{p}\right)}\left|c_{j l}\right|^{p}\right)^{\frac{1}{p}}=\left(\sum_{l=1}^{M_{j}}\left|c_{j l}\right|^{p}\right)^{\frac{1}{p}}
$$

and

$$
\begin{aligned}
\left\|f_{j}^{a} \mid L_{p}(\Gamma)\right\| & =\left(\int_{\Gamma}\left|f_{j}^{a}(x)\right|^{p} \mu(d x)\right)^{1 / p} \\
& \sim 2^{-j\left(s-\frac{n}{p}\right)}\left(\sum_{l=1}^{M_{j}}\left|c_{j l}\right|^{p} \int_{\Gamma} k^{p}\left(2^{j a}\left(x-\gamma_{j, l}\right)\right) \mu(d x)\right)^{1 / p} \\
& \sim 2^{-j\left(s-\frac{n}{p}\right)}\left(\sum_{l=1}^{M_{j}}\left|c_{j l}\right|^{p} \int_{\Gamma \cap B^{a}\left(\gamma_{j, l}, c^{-j}\right)} k^{p}\left(2^{j a}\left(x-\gamma_{j, l}\right)\right) \mu(d x)\right)^{1 / p} \\
& \geq c 2^{-j\left(s-\frac{n}{p}\right)} 2^{-j \frac{d}{p}}\left(\sum_{l=1}^{M_{j}}\left|c_{j l}\right|^{p}\right)^{1 / p}
\end{aligned}
$$

using our assumption (27) in the last estimate. Hence

$$
\left\|f_{j}^{a} \mid L_{p}(\Gamma)\right\| \geq c 2^{-j\left(s-\frac{n}{p}\right)} 2^{-\frac{j d}{p}} \quad \text { if } \quad\left\|f_{j}^{a} \mid B_{p}^{s, a}\right\| \sim 1 .
$$

Now let $T$ be an arbitrary linear operator,

$$
T: \quad B_{p}^{s, a} \hookrightarrow L_{p}(\Gamma) \quad \text { with } \quad \operatorname{rank} T \leq M_{j}-1 .
$$


Then we can find a function $f_{j}^{a}$ according to (66) with norm 1 in $B_{p}^{s, a}$ and $T f_{j}^{a}=0$. Consequently, by (67) and (68),

$$
\begin{aligned}
\left\|\operatorname{tr}_{\Gamma}-T\right\| & =\sup \left\{\left\|\left(\operatorname{tr}_{\Gamma}-T\right) f\left|L_{p}(\Gamma)\|:\| f\right| B_{p}^{s, a}\right\| \sim 1\right\} \\
& \geq\left\|\left(\operatorname{tr}_{\Gamma}-T\right) f_{j}^{a} \mid L_{p}(\Gamma)\right\| \\
& =\left\|f_{j}^{a} \mid L_{p}(\Gamma)\right\| \\
& \geq c 2^{-j\left(s-\frac{n}{p}\right)-j \frac{d}{p}} .
\end{aligned}
$$

As this is true for all $T$ according to (69), we obtain

$$
\begin{aligned}
a_{M_{j}}\left(\operatorname{tr}_{\Gamma}\right) & =\inf \left\{\left\|\operatorname{tr}_{\Gamma}-T\right\|: \operatorname{rank} T \leq M_{j-1}\right\} \\
& \geq c 2^{-j\left(s-\frac{n}{p}\right)-j \frac{d}{p}} .
\end{aligned}
$$

For $k \in \mathbb{N}$ there are numbers $j_{k} \in \mathbb{N}$ such that

$$
2^{j_{k} d} \sim k \quad \text { with } \quad j_{k_{1}} \leq j_{k_{2}} \leq \cdots \leq j_{k_{n}} \leq \cdots
$$

inserted in (68) we obtain

$$
a_{k}\left(\operatorname{tr}_{\Gamma}\right) \geq c 2^{j_{k}\left(s-\frac{n}{p}\right)} k^{-\frac{1}{p}} \geq c^{\prime} k^{\frac{1}{d}\left(\frac{n}{p}-s\right)-\frac{1}{p}},
$$

i.e., the left-hand side of the estimate (28).

Acknowledgements. I wish to thank Professor Hans Triebel and Dr. Dorothee D. Haroske for many inspiring discussions.

\section{References}

[1] O. V. Besov, V. P. Il'in, and S. M. Nikol'skiı̌, Integralnye predstavleniya funktsii i teoremy vlozheniya, Izdat. "Nauka", Moscow, 1975.

[2] S. Dachkovski, Anisotropic function spaces and related semi-linear hypoelliptic equations, Math. Nachr. 248/249 (2003), 40-61.

[3] P. Dintelmann, On Fourier multipliers between anisotropic weighted function spaces, Ph.D. thesis, TH Darmstadt, 1995.

[4] D. E. Edmunds and H. Triebel, Function spaces, entropy numbers, differential operators, Cambridge Tracts in Mathematics, vol. 120, Cambridge University Press, Cambridge, 1996.

[5] D. E. Edmunds and W. D. Evans, Spectral theory and differential operators, Oxford Mathematical Monographs, The Clarendon Press Oxford University Press, New York, 1987.

[6] K. J. Falconer, The geometry of fractal sets, Cambridge Tracts in Mathematics, vol. 85, Cambridge University Press, Cambridge, 1986.

[7] W. Farkas, Atomic and subatomic decompositions in anisotropic function spaces, Math. Nachr. 209 (2000), 83-113. 
[8] _ Eigenvalue distribution of some fractal semi-elliptic differential operators, Math. Z. 236 (2001), no. 2, 291-320.

[9] D. D. Haroske and E. Tamási, Wavelet frames for distributions in anisotropic Besov spaces, Georgian Math. J. 12 (2005), no. 4, 637-658.

[10] J. Johnsen, Pointwise multiplication of Besov and Triebel-Lizorkin spaces, Math. Nachr. 175 (1995), 85-133.

[11] S. M. Nikol'skiǔ, Priblizhenie funktsii mnogikh peremennykh i teoremy vlozheniya, "Nauka", Moscow, 1977.

[12] T. Runst and W. Sickel, Sobolev spaces of fractional order, Nemytskij operators, and nonlinear partial differential equations, de Gruyter Series in Nonlinear Analysis and Applications, vol. 3, Walter de Gruyter \& Co., Berlin, 1996.

[13] H.-J. Schmeisser and H. Triebel, Topics in Fourier analysis and function spaces, A WileyInterscience Publication, John Wiley \& Sons Ltd., Chichester, 1987.

[14] E. M. Stein and S. Wainger, Problems in harmonic analysis related to curvature, Bull. Amer. Math. Soc. 84 (1978), no. 6, 1239-1295.

[15] B. Stöckert and H. Triebel, Decomposition methods for function spaces of $B_{p, q}^{s}$ type and $F_{p, q}^{s}$ type, Math. Nachr. 89 (1979), 247-267.

[16] H. Triebel, Fourier analysis and function spaces (selected topics), Teubner Verlagsgesellschaft, Leipzig, 1977.

[17] _ Theory of function spaces, Monographs in Mathematics, vol. 78, Birkhäuser Verlag, Basel, 1983.

[18] _ Theory of function spaces. II, Monographs in Mathematics, vol. 84, Birkhäuser Verlag, Basel, 1992.

[19] _ Fractals and spectra, Monographs in Mathematics, vol. 91, Birkhäuser Verlag, Basel, 1997.

[20] — The structure of functions, Monographs in Mathematics, vol. 97, Birkhäuser Verlag, Basel, 2001.

[21] __ Approximation numbers in function spaces and the distribution of eigenvalues of some fractal elliptic operators, J. Approx. Theory 129 (2004), no. 1, 1-27.

[22] M. Yamazaki, A quasihomogeneous version of paradifferential operators. I. Boundedness on spaces of Besov type, J. Fac. Sci. Univ. Tokyo Sect. IA Math. 33 (1986), no. 1, 131-174. 